Cahiers Charlevoix

Études franco-ontariennes
Cahiers Charlevoix Études franco-ontariennes

or Crevenerix of

\title{
Langue maternelle et langue d'usage dans les foyers mixtes francophones : les enjeux de l'exogamie
}

\section{Roger Bernard}

Volume 1, 1995

URI : https://id.erudit.org/iderudit/1039464ar

DOI : https://doi.org/10.7202/1039464ar

Aller au sommaire du numéro

\section{Éditeur(s)}

Société Charlevoix

Presses de l’Université d'Ottawa

ISSN

1203-4371 (imprimé)

2371-6878 (numérique)

Découvrir la revue

Citer cet article

Bernard, R. (1995). Langue maternelle et langue d'usage dans les foyers mixtes francophones : les enjeux de l'exogamie. Cahiers Charlevoix, 1, 241-289.

https://doi.org/10.7202/1039464ar
Résumé de l'article

La dilution de l'identité est aussi à l'ordre du jour des travaux du sociologue Roger Bernard de l'Université d'Ottawa. La minorisation de l'élément canadien-français, passé du village à la ville, a favorisé, outre la bilinguisation et l'anglicisation, l'exogamie dont les enjeux linguistiques révèlent une situation inquiétante : même à la maison, l'auteur enregistre la très nette force d'attraction de l'anglais comme langue d'usage dans les foyers mixtes composés d'un parent francophone et de son conjoint anglophone et, fait remarquable qui bouscule le concept même de langue maternelle, la mère francophone ne réussit pas mieux que le père francophone à transmettre le français dans ce contexte. Ces recherches débordent le cadre de l'Ontario et ont des retombées sur tout le Canada français. 


\section{LANGUE MATERNELLE ET LANGUE D'USAGE DANS LES FOYERS MIXTES FRANCOPHONES: LES ENJEUX DE L'EXOGAMIE}

Roger Bernard

Faculté d'éducation

Université d'Ottawa, Ottawa

Cahiers Charlevoix 1, 1995, pp. 241-289. 


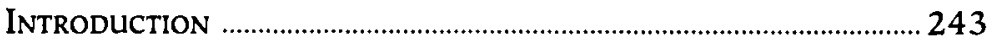

1. LE CONTEXTE DE LA MONTÉE DE L'EXOGAMIE ………...................245

2. EXOGAMIE, ANGLICISATION ET MINORISATION ...............................254

3. LA TRANSMISSION DE LA LANGUE MATERNELLE

A L'INTÉRIEUR DES FAMILLES EXOGAMES FRANCOPHONES ............ 262

3.1. Méthodologie de l'enquête

3.1.1. La méthodologie de sélection des répondants

3.1.2. L'échantillon des familles exogames francophones

3.1.3. Les tests d'hypothèses

3.2. La force d'attraction respective des langues française et anglaise

3.3. La force de rétention des parents respectifs Récapitulation

4. LaNGUe d'USAGe et LaNGUe PRINCIPALE

DANS LES FOYERS EXOGAMES

4.1. La force d'attraction respective des langues anglaise et française

4.2. La force de rétention des parents respectifs Récapitulation

4.3. Langue d'usage à la maison

Récapitulation

ConcLusion

ANNEXE 1. HyPOTHĖSES EN REGARD DE LA TRANSMISSION

DE LA LANGUE MATERNELLE

ANNEXe 2. Hypothèses en Regard de la langue PRINCIPALE .......285

ANNEXe 3. Hypothèses en REgard de La langue d'usage $A U$ FOYER 


\section{LANGUE MATERNELLE ET LANGUE D'USAGE DANS LES FOYERS MIXTES FRANCOPHONES: LES ENJEUX DE L'EXOGAMIE}

\section{INTRODUCTION}

Si au Canada les taux de mariages exogames et endogames varient considérablement d'un groupe ethnique à l'autre, l'endogamie représente encore aujourd'hui la norme pour la très grande majorité des Canadiens et des Canadiennes. La taille du groupe ethnique ou son poids démographique sont des facteurs explicatifs importants de ces variations: habituellement plus le groupe est grand et son poids démographique imposant, plus le taux d'endogamie sera élevé. Les taux d'exogamie des époux et des épouses de langue française au Canada illustrent le bien-fondé de cette hypothèse: ce taux est très faible au Québec, faible au Nouveau-Brunswick, élevé en Ontario et très élevé en Colombie-Britannique (tableau 1).

Depuis la fin des années soixante, l'exogamie est devenue un phénomène social qui caractérise l'évolution des communautés francophones et 
acadiennes $^{1}$ qui se trouvent en situation minoritaire au Canada. Pour bien comprendre l'importance de l'exogamie qui se trouve au cour même de la francophonie, il faut d'abord la replacer dans l'ensemble des facteurs démographiques qui en favorisent la montée (certains diront qu'il y a là une forme de déterminisme démographique), découvrir les changements de valeurs qui la légitiment et analyser les conditions qui en encouragent l'expansion comme forme d'organisation familiale.

Dans les enjeux de l'exogamie, plusieurs analystes considèrent qu'il s'agit effectivement d'une situation inquiétante pour l'avenir des communautés francophones et acadiennes. Mais avant d'en arriver à ce diagnostic pessimiste, nous devons franchir plusieurs étapes. En premier lieu, nous devons étudier le contexte de la montée de l'exogamie, les sentiments d'appartenance et les processus de socialisation qui se développent à l'intérieur des familles exogames pour voir si les mariages mixtes créent des conditions et un contexte qui conduisent aux transferts linguistiques et à l'assimilation culturelle; en deuxième lieu, nous devons essayer de comprendre l'importance du rôle de la famille exogame en regard du maintien de la vitalité linguistique et culturelle des communautés francophones et acadiennes au Canada, et ensuite voir quels sont les liens entre l'exogamie, la minorisation et l'anglicisation; en troisième lieu, nous pourrons tenter de répondre à la question cruciale qui constitue l'objectif premier de cette analyse: estce que l'exogamie est un phénomène inquiétant pour

\footnotetext{
${ }^{1}$ NDLR - L'expression "francophone et acadien" désigne les communautés de langue française vivant dans les provinces anglaises du Canada, selon la terminologie étonnante adoptée par la «Fédération des communautés francophones et acadienne du Canada» (FCFAC).
} 
la survie et le développement des communautés francophones et acadiennes qui se trouvent en situation minoritaire au Canada? Est-ce que l'exogamie est une cause importante de la bilinguisation, de l'assimilation et de l'anglicisation des jeunes francophones du Canada français? Pour ce faire, nous étudierons les processus de transmission de la langue maternelle à l'intérieur des foyers exogames et nous examinerons les capacités de maintien du français comme langue d'usage dans ces mêmes familles.

Pour bien amorcer notre analyse et notre réflexion, il faut revoir le contexte de la montée de l'exogamie et dévoiler la conjoncture qui en favorise l'institutionnalisation.

\section{Le conteXte de la montée de l'eXogamie}

À l'époque de la société canadienne-française traditionnelle et catholique, celle qui est caractérisée par son mode de vie rurale, l'Église et la famille sont les principales institutions qui assurent la vitalité linguistique du Canada français et qui assument la responsabilité de la transmission de la culture canadienne-française. Il faut être bien conscient qu'à cette époque, les Canadiens-Français ${ }^{2}$ de l'ensemble du Canada (le Québec inclus) forment une société relativement homogène, que les distinctions identitaires provinciales sont négligeables et que

\footnotetext{
${ }^{2}$ NDLR - Contrairement à l'usage courant qui veut qu'on écrive Canadiens français, nom propre, et canadiens-français, adjectif, l'auteur propose, pour une raison idéologique, un changement dont il s'explique ainsi: «La création des noms propres composés CanadiensFrançais et Canadiens-Anglais, dont la formation est conforme au génie de la langue française, marque l'appartenance à la fois nationale et ethnique compte tenu de la dualité linguistique et culturelle du Canada.»
} 
la religion catholique est à la base de la culture canadienne-française. Si la religion représente la valeur centrale de la culture et l'élément fondamental de l'identité, nous devons en même temps reconnaître que la foi catholique était gardienne de la langue française et qu'en retour le maintien de la langue favorisait le maintien de la foi. La langue et la foi sont deux éléments de l'univers culturel qui sont étroitement liés l'un à l'autre formant le noyau dur de la culture canadienne-française: les luttes pour la défense de la langue française recouvrent très souvent un autre combat, celui du témoignage de la fidélité à la religion catholique.

Dans ce contexte culturel et religieux, l'exogamie est pratiquement impensable: choisir un conjoint de l'autre groupe, un Canadien-Anglais, c'est prendre le risque de perdre sa culture et sa foi. En effet, dans l'univers des représentations symboliques, les classifications sont claires et nettes: le français est automatiquement associé au catholicisme et l'anglais, au protestantisme; très souvent l'autre sera identifié comme un "protestant", même si l'identification est essentiellement linguistique. Dans cet univers religieux, l'exogamie s'apparente à la transgression d'un tabou. Les permissions des parents pour se marier à l'extérieur du groupe sont difficiles à obtenir et les embûches de l'Église, difficiles à surmonter; de plus, les futurs époux risquent la marginalisation et l'exclusion sociale. Les barrières culturelles et religieuses deviennent des empêchements qui limitent sérieusement le nombre de mariages mixtes; à cette époque, l'endogamie est une norme sociale contraignante qui est transmise par la famille et l'Église dans des actions et des enseignements conjoints: les 
freins à l'exogamie sont à la fois sociaux, culturels et institutionnels. Les empêchements touchent toutes les facettes de la vie de la société canadiennefrançaise. À cette époque, il n'est pas facile de se marier à l'extérieur de son groupe.

Les facteurs explicatifs en regard de la force de l'endogamie comme norme sociale comprennent aussi des phénomènes démographiques et écologiques. En effet, très souvent les Canadiens-Français sont majoritaires dans les villages et les paroisses qu'ils habitent, même s'ils sont très minoritaires lorsque nous considérons l'ensemble de la population des différentes provinces canadiennes, à l'exception du Québec et du Nouveau-Brunswick. Dans les villes, ils se regroupent autour d'une paroisse et, de ce fait, ils arrivent à former des quartiers où ils sont plus ou moins minoritaires. L'école primaire de rang, de village ou de quartier catholique et bilingue, rassemble une population écolière relativement homogène composée essentiellement de jeunes canadiens-français catholiques. Au secondaire, les choses se compliquent, mais la fréquentation est limitée et une bonne proportion de ceux qui fréquentent l'école secondaire se retrouvent dans des collèges ou des couvents privés, catholiques et français, les autres étant répartis dans les high schools publics et anglais où les probabilités de fréquentations "exogames» sont plus élevées. Comme nous pouvons le constater, ces phénomènes sociodémographiques et écologiques s'ajoutent aux facteurs culturels et institutionnels pour favoriser le maintien de l'endogamie comme norme et pratique sociales.

Le dernier facteur que nous avons retenu est lié aux contacts restreints entre les Canadiens-Français 
et les membres des autres groupes culturels et religieux. Il n'y a pas si longtemps, les différentes communautés linguistiques étaient littéralement enfermées dans leur unilinguisme respectif; les contacts entre les groupes étaient très limités parce que la grande majorité des Canadiens de langue anglaise ne connaissaient pas le français et parallèlement une proportion imposante de Canadiens-Français ne connaissaient pas l'anglais.

Les changements culturels, religieux, démographiques, écologiques et sociolinguistiques des dernières décennies nous amènent à une tout autre réalité. Le passage d'une société traditionnelle, rurale et catholique à une société moderne, urbaine et industrielle, a modifié presque complètement le milieu de vie des communautés francophones et acadiennes, surtout celles qui se trouvent en situation minoritaire au Canada. Les communautés majoritaires du Québec et du Nouveau-Brunswick ne connaîtront pas des changements de milieu de vie aussi dramatiques: de façon générale, nous pouvons affirmer que plus les communautés seront minoritaires, plus les changements seront profonds et déterminants.

La transition du monde rural et agricole à un monde urbain et industriel exige que bon nombre de Canadiens-Français quittent les villages, les paroisses et les quartiers où ils étaient majoritaires pour aller s'installer dans des villes, des paroisses et des quartiers où ils deviennent minoritaires; il en est ainsi pour les écoles et les autres institutions qu'ils fréquentent. Étant bilingues, ils ne recherchent pas le quartier francophone à tout prix. Plusieurs parents se voient contraints d'envoyer leurs enfants à 
l'école anglaise. Pour une grande majorité des francophones, le processus de modernisation s'accompagne presque toujours d'un processus de minorisation et plus le processus de modernisation avance, plus le processus de minorisation s'accentue. Les statistiques des recensements des trois dernières décennies montrent clairement la progression de la minorisation ${ }^{3}$. L'hétérogénéité remplace l'homogénéité. Le contact est maintenant continuel avec les anglophones; la langue anglaise domine le monde du travail, des affaires, des communications et des services. Dans ce nouvel entourage, la sphère réservée au français se limite trop souvent à la vie familiale, alors que la langue anglaise s'impose dans l'ensemble des activités de la vie quotidienne. C'est dans ce contexte que les francophones minoritaires doivent absolument être bilingues s'ils veulent se tailler une place; la situation pratique l'exige.

Comme suite à ces grands changements, nous pouvons facilement voir que le nouvel environnement social et culturel va favoriser l'exogamie: les francophones sont de plus en plus minoritaires, de plus en plus dispersés et de plus en plus bilingues, trois conditions qui vont expliquer, en partie, l'augmentation des taux d'exogamie.

Le délestage du mode de pensée traditionnel et religieux fait en sorte que la religion catholique, élément central de la culture traditionnelle, est lentement détachée des luttes pour la survie de la langue et de la culture françaises. Les adages «la langue gardienne de la foi» et «la foi gardienne de la langue»

\footnotetext{
${ }^{3}$ Roger Bernard, Le Déclin d'une culture. Recherche, analyse et bibliographie. Francophonie hors Québec, 1980-1990, Ottawa, Fédération des jeunes Canadiens français, 1990, 198 p.
} 
perdent sens et pertinence. La francité et le bilinguisme deviennent des valeurs qui remplacent la religion en tant que valeur dominante. L'appartenance sociétale traditionnelle, celle du Canada français, cède la place à une identité plus régionale et plus linguistique; les communautés francophones et acadiennes succèdent à la société canadiennefrançaise; les combats pour la survie de la langue et de la culture françaises passent par l'axe du bilinguisme qui devient effectivement l'élément central de la culture collective et de l'identité individuelle. L'idéal est de connaître l'anglais, de l'écrire et de le parler comme les anglophones, ou encore mieux qu'eux, sans succomber au transfert linguistique et à l'anglicisation, alors que le contexte conduit naturellement à l'intégration sociétale et à l'assimilation à la majorité.

Les barrières culturelles, religieuses et linguistiques sont tombées; les facteurs démographiques, écologiques et sociaux favorisent maintenant une cohabitation plus poussée et un partage des institutions sociales. L'exogamie est une réalité avec laquelle les communautés francophones et acadiennes doivent maintenant composer.

Est-ce que ces valeurs, ces situations et ces conditions risquent de changer sensiblement durant les prochaines années? La réponse est simple et directe: non! Au contraire, les tendances démographiques vont fort probablement s'accentuer durant les prochaines décennies et créer un contexte qui va contribuer à une augmentation des taux d'exogamie chez les francophones; de fait, plus les francophones seront minoritaires et dispersés, plus le taux d'exogamie sera élevé. 
En quelques décennies, les communautés francophones et acadiennes passent d'une culture religieuse très contraignante et hermétique à une culture médiatique éclatée et ouverte. L'exogamie n'est plus un tabou; au contraire, elle favorise une cohabitation plus engagée, permet de vivre une situation de bilinguisme intégral et de réaliser le grand rêve de nombreux francophones qui se définissent par leur bilinguisme et leur appartenance à deux cultures.

En considérant que les phénomènes démographiques sont relativement stables et que les tendances à la hausse ou à la baisse sont très difficiles à renverser, nous pourrions, en analysant les données démographiques des dernières décennies, brosser le tableau suivant pour les prochaines années:

- la minorisation et la dispersion des francophones risquent de s'accentuer au Canada;

- les transferts linguistiques du français vers l'anglais vont augmenter; de fait, plus le milieu sera minoritaire, plus les taux de transferts linguistiques seront élevés;

- il n'y a pas de signe pour prédire que les indices de fécondité de la population de langue maternelle française vont augmenter; ils demeureront faibles et inférieurs à la moyenne canadienne;

- le nombre de jeunes francophones va continuer de diminuer; le recul sera plus prononcé dans les milieux très minoritaires;

- la population francophone hors Québec vieillit: les pyramides d'âge s'inversent; plus les francophones sont minoritaires, plus le vieillissement se fait sentir et plus la proportion de jeunes francophones diminue. 
Dans ce contexte, il est inévitable que nous assistions à une montée de l'exogamie, et il serait tout à fait inattendu que dans un avenir rapproché nous puissions observer une régression de ce phénomène. Le tableau 1 illustre l'ampleur de l'exogamie des époux et des épouses de langue maternelle française dans les différentes provinces canadiennes, en 1971, en 1986 et en 1991. Les données montrent clairement qu'il y a une hausse significative des taux d'exogamie entre 1971 et 1991 et que ces taux sont sensiblement les mêmes, qu'il s'agisse des époux ou des épouses de langue maternelle française (1986). En 1991, nous retrouvons une proportion plus élevée de mariages hétérolinguistiques que de mariages homolinguistiques dans les provinces ou régions suivantes: Colombie-Britannique, Alberta, Saskatchewan, Terre-Neuve, Yukon et les Territoires du Nord-Ouest. Chez les francophones de l'Ontario, nous relevons un taux relativement élevé d'exogamie, alors qu'il est plutôt faible au Nouveau-Brunswick et très faible au Québec: plus le poids démographique d'un groupe culturel est faible, plus son taux d'exogamie est élevé.

Les données et les tendances qui se dégagent du premier tableau sont sans équivoque: l'exogamie est aujourd'hui une réalité sociale avec laquelle les communautés francophones et acadiennes doivent traiter; en outre, dans les milieux très minoritaires, il ne serait pas surprenant de constater que la majorité des nouveaux mariages des époux et des épouses de langue maternelle française soient des mariages mixtes. Avis aux communautés francophones: l'exogamie est là pour rester; il s'agit effectivement d'une nouvelle forme d'organisation familiale; nous assistons à l'institutionnalisation de l'exogamie. 


\section{TABLEAU 1}

Taux d'exogamie des époux et des épouses de langue maternelle française, provinces et Canada, 1971, 1986 et 1991.

\begin{tabular}{|c|c|c|c|c|c|}
\hline \multirow[b]{2}{*}{ Provinces } & \multicolumn{2}{|c|}{$\begin{array}{c}\text { Epoux de langue } \\
\text { maternelle } \\
\text { française }\end{array}$} & \multicolumn{2}{|c|}{$\begin{array}{c}\text { Epouse de langue } \\
\text { maternelle } \\
\text { française }\end{array}$} & \multirow{2}{*}{$\begin{array}{c}\text { Époux et épouse } \\
\text { de langue } \\
\text { maternelle } \\
\text { française } \\
1991\end{array}$} \\
\hline & 1971 & 1986 & 1971 & 1986 & \\
\hline C. $-\mathrm{B}$. & 61,1 & 67,6 & 58,5 & 68,1 & 71,2 \\
\hline Alberta & 47,3 & 55,9 & 47,6 & 57,9 & 61,5 \\
\hline Sask. & 42,3 & 53,0 & 42,0 & 54,7 & 57,8 \\
\hline Manitoba & 32,4 & 41,0 & 32,8 & 40,4 & 45,6 \\
\hline Ontario & 28,9 & 37,8 & 30,4 & 34,4 & 40,9 \\
\hline Québec & - & 3,4 & - & 2,7 & - \\
\hline N.-B. & 9,1 & 11,3 & 10,9 & 10,8 & 13,7 \\
\hline Î.-P.-E. & 23,6 & 39,5 & 28,7 & 37,3 & 43,5 \\
\hline N. $-\hat{E}$. & 32,6 & 39,6 & 32,1 & 40,5 & 45,3 \\
\hline T. $-\mathrm{N}$. & 45,5 & 57,9 & 38,0 & 61,5 & 63,9 \\
\hline T.N.-O. & - & 58,2 & - & 65,7 & 63,8 \\
\hline Yukon & - & 60,7 & - & 69,4 & 67,0 \\
\hline $\begin{array}{l}\text { Canada san } \\
\text { le Québec }\end{array}$ & 一 & 34,8 & - & 33,1 & 38,3 \\
\hline Canada & 一 & 8,2 & - & 7,3 & - \\
\hline
\end{tabular}

SOURCES ${ }^{4}$

En 1986, seuls les mariages avec un partenaire ayant déclaré l'anglais ou une autre langue comme seule langue matemelle ont été considérés.

Pour bien saisir l'ampleur et les conséquences sociales et linguistiques de l'exogamie, il faut, dans

${ }^{4}$ Roger Bernard, Le Choc des nombres. Dossier statistique sur la francophonie canadienne, 1951-1986, Ottawa, Fédération des jeunes Canadiens français, 1990, 311 p.; L. Dallaire et Réjean Lachapelle, Profils linguistiques des communautés minoritaires de langue officielle, Ottawa, Secrétariat d'État du Canada, 1990 (13 volumes); Fédération des francophones hors Québec, Les Héritiers de Lord Durham, vol. 1, 1977 , 125 p.; Réjean Lachapelle, "L'Exogamie dans les populations francophones en situation minoritaire», communication au colloque national sur l'exogamie, Rockland (Ontario), février 1994. 
un premier temps, tenter de répondre à la question cruciale, est-ce que l'exogamie est un phénomène inquiétant en regard de la vitalité linguistique et culturelle des communautés francophones et acadiennes, et dans un deuxième temps, analyser les comportements langagiers à l'intérieur des familles mixtes francophones.

\section{EXOGAMIE, ANGLICISATION ET MINORISATION}

$\mathrm{Si}$, aujourd'hui, plus du tiers des époux et des épouses de langue maternelle française qui vivent à l'extérieur du Québec se retrouvent dans une famille mixte, nous pouvons remarquer, selon les données du premier tableau, une hausse significative du taux d'exogamie entre 1971 et 1991 , et rappeler que les taux d'exogamie varient considérablement d'une province à l'autre, mais que cette variation est en très grande partie due au poids démographique des francophones. En 1991, le taux d'exogamie des époux et des épouses de langue française de la ColombieBritannique était de 71,2\%, alors qu'il était de 13,7 \% pour les époux et épouses francophones du NouveauBrunswick. Cette même année, dans les provinces de la Colombie-Britannique, de l'Alberta, de la Saskatchewan et de Terre-Neuve, ainsi que dans les Territoires du Nord-Ouest et le Yukon, nous retrouvons une proportion plus élevée de familles hétérolinguistiques que de familles homolinguistiques. Entre 1981 et 1991 , les tendances se maintiennent et l'exogamie progresse: plus de la moitié des francophones qui vivent à Terre-Neuve ou dans les provinces situées à l'ouest du Manitoba ont un conjoint dont la langue maternelle n'est pas le français ${ }^{5}$. Dans

${ }^{5}$ Réjean Lachapelle, «L'Exogamie dans les populations francophones 
toutes ces provinces, les francophones sont très minoritaires: ils représentent moins de $3 \%$ de la population générale. À l'île-du-Prince-Édouard, en Nouvelle-Écosse, en Ontario et au Manitoba, dans les provinces où la proportion de francophones varie entre 4 et $5 \%$, le taux d'exogamie fluctue de 40 à $50 \%^{6}$. Au Nouveau-Brunswick, où les francophones forment le tiers de la population, il frôle le $15 \%$, une augmentation substantielle par rapport à 1986. Les tendances qui ne démordent pas depuis plusieurs décennies confirment l'hypothèse suivante: le taux d'exogamie chez les francophones est inversement proportionnel à leur poids démographique; en situation très minoritaire, l'exogamie est omniprésente, en situation majoritaire, quasi absente.

Devant ces réalités démographiques dures, il faut revenir à la question cruciale, est-ce que l'exogamie est un phénomène inquiétant en regard de la vitalité linguistique et culturelle des communautés francophones minoritaires? Dans son analyse de l'exogamie dans les populations francophones en situation minoritaire, Réjean Lachapelle, en $1994^{7}$, présente la vue d'ensemble suivante des données du recensement de 1991:

- «La fréquence de l'exogamie chez les francophones est inversement liée à la proportion qu'ils représentent dans le milieu.

- Le taux d'anglicisation est fonction de la fréquence de l'exogamie dans le milieu.

- L'exogamie est en augmentation chez les jeunes

en situation minoritaire», op. cit.

${ }^{6}$ Ibid.

7 Ibid. 
francophones, mais l'anglicisation a plutôt tendance à diminuer.

- La transmission de la mère aux enfants du français langue maternelle est fonction des attributs linguistiques du père.»

Dans un premier temps, Lachapelle démontre clairement que le taux d'exogamie des francophones est inversement proportionnel à leur poids démographique et que cette relation devient exponentielle lorsque la proportion de francophones dans les divisions de recensement est inférieure à $10 \%$. La démonstration est aussi éloquente lorsqu'il soutient que le taux d'anglicisation des francophones est fonction de la fréquence de l'exogamie dans le milieu de vie dans une relation presque linéaire qui permet d'affirmer que chaque unité d'augmentation du taux d'exogamie (au Canada sans le Québec) est accompagnée d'une augmentation proportionnelle du taux net de transfert linguistique. Il est évident que ces trois phénomènes démographiques, notamment l'exogamie, la minorisation et l'anglicisation, sont fortement corrélés, mais les analystes ne les ont pas introduits dans un diagramme de causalité pour établir la pondération de chaque phénomène en regard de l'assimilation.

Toutefois, dans un deuxième temps, Lachapelle établit que la transmission du français langue maternelle varie sensiblement d'une situation d'endogamie à une situation d'exogamie. En effet, lorsque les deux parents sont francophones, la transmission du français langue maternelle aux enfants se fait dans $90 \%$ des cas, alors que cette proportion baisse à $20 \%$ lorsque la mère est francophone et que le père est 
anglophone. Cette proportion augmente considérablement si l'époux anglophone peut parler le français: dans ce cas la transmission du français langue maternelle de l'épouse aux enfants se produit dans la moitié des situations.

Pour bien comprendre les relations causales qui relient l'exogamie et l'assimilation linguistique chez les minorités canadiennes-françaises, nous ne pouvons passer à coté des nombreuses et excellentes études statistiques présentées par Charles Castonguay ${ }^{8}$, études qui complètent très bien celles de Réjean Lachapelle.

Castonguay et Lachapelle établissent clairement que les taux d'exogamie et de transferts linguistiques augmentent à travers le temps et les générations, qu'ils sont fortement corrélés et qu'ils sont inversement proportionnels au poids démographique des différentes minorités provinciales canadiennesfrançaises. Les données analysées et présentées dans le Choc des nombres en 1991 corroborent les conclusions de Castonguay et Lachapelle. Si Castonguay ${ }^{9}$ reconnaît qu'il y a une forte relation entre l'exogamie et l'anglicisation, il invite à la prudence eu égard aux

${ }^{8}$ Charles Castonguay, "Les Transferts linguistiques au foyer», $R e$ cherches sociographiques, vol. XVII, $n^{\circ} 3$, 1976, pp. 341-351; id., «Les Mécanismes du transfert linguistique", Cahiers québécois de démographie, vol. 6, $\mathrm{n}^{\circ} 3$ spéclal, 1977, pp. 138-155; id., «Exogamie et anglicisation chez les minorités canadiennes-françaises», La Revue canadienne de sociologie et d'anthropologie, vol. $16, \mathrm{n}^{\circ} 1,1979$, pp. 21 31 ; id., L'Evolution de l'exogamie et de ses incidences sur les transferts linguistiques chez les populations provinciales de langue maternelle française au Canada entre 1971 et 1981 , Québec, Conseil de la langue française, 1983.

${ }^{9}$ Charles Castonguay, «Exogamie et anglicisation chez les minorités canadiennes-françaises", op. cit., pp. 21-31; id., L'Évolution de l'exogamie [...], op. cit. 
relations de causalité: l'exogamie est une cause importante des transferts linguistiques, mais il ne s'agit pas de l'unique cause immédiate de l'anglicisation. Si au Québec, moins de la moitié des transferts linguistiques s'explique par l'exogamie, en considérant que les taux d'exogamie et de transferts sont faibles, force est de reconnaître que les personnes exogames comptent généralement pour moins de la moitié des transferts linguistiques chez les minorités francophones à l'extérieur du Québec, et ça malgré les taux très élevés d'exogamie et d'anglicisation. Plus les francophones et les Acadiens sont minoritaires, plus l'exogamie prend une "valeur explicative dégressive» en regard de l'assimilation. Souvent, c'est le transfert linguistique qui précède le mariage mixte, ou qui conduit à l'exogamie.

Nonobstant les remarques et les nuances qui précèdent, Castonguay reconnaît, comme de nombreux analystes, que le mariage mixte est un initiateur "primordial du transfert à l'anglais comme langue au foyer» ${ }^{10}$ et plus particulièrement chez les minorités les plus résistantes à l'anglicisation. Plus les francophones sont minoritaires, plus l'anglais s'imposera comme langue d'usage dans les foyers mixtes: en Colombie-Britannique, en Alberta, en Saskatchewan, au Manitoba et à l'île-du-Prince-Édouard, près de $100 \%$ des époux et des épouses de langue maternelle française s'anglicisent dans les foyers exogames; en Ontario, en Nouvelle-Écosse et à Terre-Neuve, ces taux d'anglicisation varient de 79,6 à $92,3 \%$, et au Nouveau-Brunswick, de 62,0 à $70,8 \%$, selon les données du recensement de 1986 (tableau 2).

${ }^{10}$ Id., «Exogamie et anglicisation chez les minorités canadiennesfrançalses», op. cit., p. 21. 
Lorsque nous considérons l'ensemble des communautés francophones hors Québec, 9 époux sur 10 de langue maternelle française s'anglicisent en situation d'exogamie, alors que cette proportion est de 8 sur 10 pour les épouses.

\section{TABLEAU 2}

Taux d'anglicisation du partenaire de langue maternelle française dans les mariages hétérolinguistiques provinces et Canada, 1971 et 1986.

\begin{tabular}{|c|c|c|c|c|}
\hline \multirow[b]{2}{*}{ Provinces } & \multicolumn{2}{|c|}{$\begin{array}{l}\text { Époux de langue } \\
\text { maternelle française }\end{array}$} & \multicolumn{2}{|c|}{$\begin{array}{l}\text { Epouse de langue } \\
\text { maternelle française }\end{array}$} \\
\hline & 1971 & 1986 & 1971 & 1986 \\
\hline Colombie-Britannique & 97,9 & 97,4 & 94,6 & 92,9 \\
\hline Alberta & 96,8 & 97,0 & 95,7 & 93,4 \\
\hline Saskatchewan & 97,3 & 96,9 & 95,5 & 96,6 \\
\hline Manitoba & 95,1 & 94,1 & 91,6 & 92,5 \\
\hline Ontario & 92,8 & 90,0 & 87,7 & 80,1 \\
\hline Québec & - & 29,8 & - & 14,5 \\
\hline Nouveau-Brunswick & 82,6 & 70,8 & 77,5 & 62,0 \\
\hline Île-du-Prince-Édouard & 95,8 & 100,0 & 91,3 & 91,3 \\
\hline Nouvelle-Écosse & 93,6 & 92,3 & 90,2 & 86,3 \\
\hline Terre-Neuve & 91,4 & 79,6 & 86,7 & 86,5 \\
\hline Canada sans le Québec & - & 88,4 & - & 82,8 \\
\hline Canada & - & 70,3 & - & 54,2 \\
\hline
\end{tabular}

SOURCES 11

Dans les foyers mixtes, il appert que l'anglais dominera dans l'aire linguistique familiale et, naturellement, les enfants adopteront comme langue d'usage la langue des parents. Dans les mariages hétérolinguistiques où la mère est de langue maternelle française et le père de langue anglaise et qu'il parle

${ }^{11}$ Statistique Canada, recensement de 1971; recensement de 1986; Charles Castonguay, ibid.; Roger Bernard, Le Choc des nombres, op. cit., p. 222. 
l'anglais à la maison, situation habituelle, il s'ensuit que $69 \%$ des enfants parleront seulement l'anglais, $17 \%$ l'anglais et le français et $14 \%$ le français seulement (tableau 3); si le père anglophone parle français à la maison (cas très rare), la très grande majorité des enfants parleront le français seulement à la maison.

\section{TABLEAU 3}

Langues parlées à la maison par les enfants de mère de langue maternelle française et de père de langue maternelle anglaise selon la langue parlée à la maison par le père, 1986.

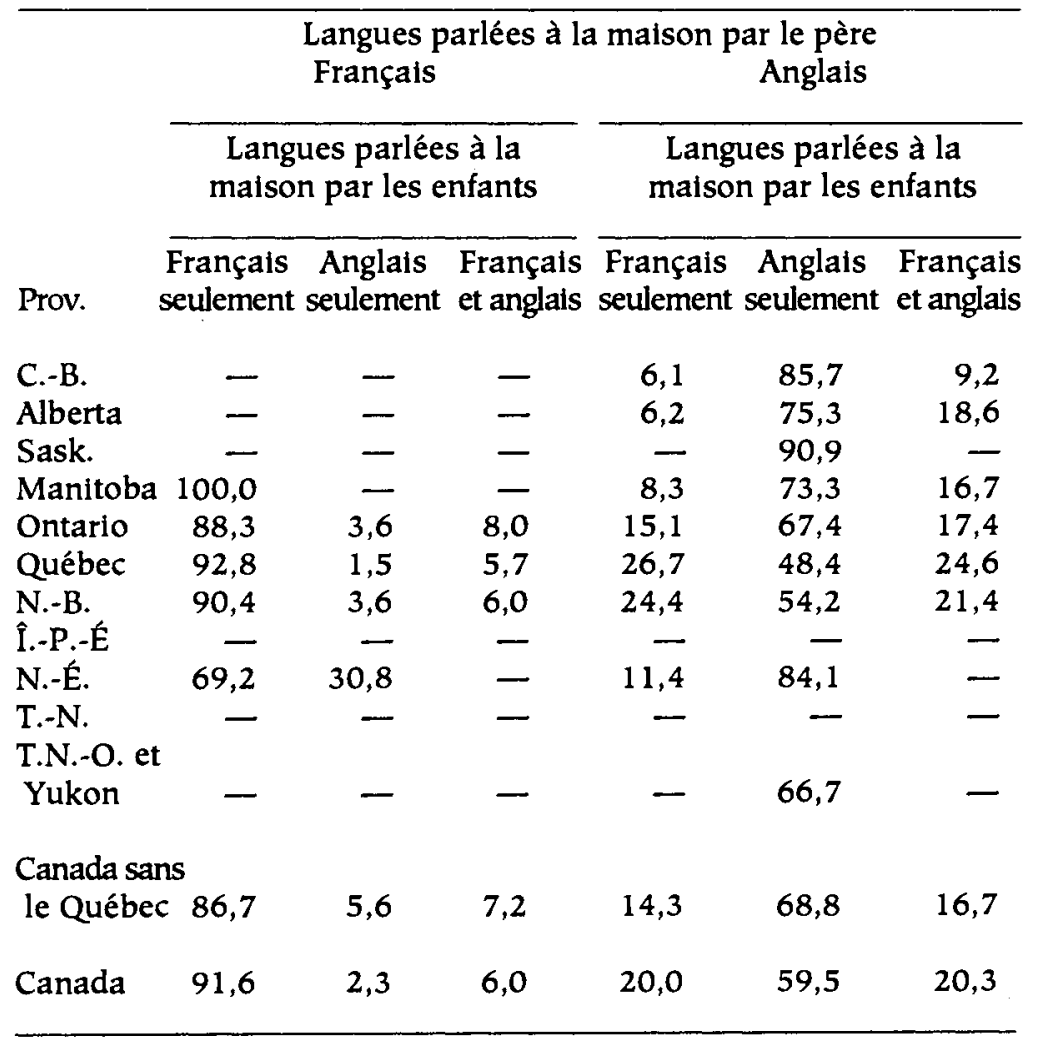

SOURCES ${ }^{12}$

${ }^{12}$ Statistique Canada, recensement de 1986; Roger Bernard, Le Choc des nombres, op. cit., p. 224. 
À la question cruciale, «est-ce que l'exogamie est un phénomène inquiétant en regard de la vitalité linguistique et culturelle des communautés francophones minoritaires?", nous sommes contraints de reconnaître, à la lecture des données statistiques et en acceptant l'analyse des spécialistes, que l'exogamie est une des causes de la bilinguisation et de l'anglicisation de ces minorités. En général, nous pouvons affirmer que l'endogamie protège la famille francophone de l'assimilation, mais il ne s'agit pas d'un rempart; dans des conditions de très faible vitalité ethnolingistique, la famille endogame ne réussit pas à stopper les transferts linguistiques. Selon cette perspective, l'exogamie est souvent considérée comme un phénomène externe à la francophonie, un lieu de passage de la minorité à la majorité.

Nous devons reprendre nos études en adoptant une nouvelle perspective, en reconnaissant une nouvelle réalité sociale. L'exogamie est là pour rester; elle est maintenant au cour de la francophonie minoritaire, et il s'agit effectivement d'une nouvelle forme d'organisation familiale. Nous devons maintenant essayer de comprendre l'exogamie comme un phénomène interne à la francophonie minoritaire. C'est dans cette perspective que nous dépouillerons les données de l'enquête sociale pan-canadienne: étudier les processus de transmission de la langue maternelle à l'intérieur des foyers exogames francophones et examiner les capacités de maintien du français comme langue d'usage dans ces mêmes familles. 
3. LA TRANSMISSION DE LA LANGUE MATERNELLE $\AA$ L'INTÉRIEUR DES FAMILLES EXOGAMES FRANCOPHONES

\subsection{MÉTHODOLOGIE DE L'ENQUÊTE}

\subsubsection{LA MÉTHODE DE SÉLECTION DES RÉPONDANTS}

L'échantillon de 758 familles exogames francophones que nous avons constitué pour la présente étude est effectivement un sous-échantillon d'une enquête sociale nationale réalisée en 1990 . Les jeunes francophones âgés de 15 à 24 ans et vivant à l'extérieur du Québec représentaient notre population cible.

Construire un échantillon représentatif des jeunes francophones qui fréquentaient les écoles, les collèges et les universités de langue française à travers le Canada n'est pas une mince affaire. Nous avions un autre critère de représentativité important: le degré de vitalité ethnolinguistique du milieu de vie des jeunes francophones. De nombreux problèmes de méthodologie et de logistique ont surgi tout au long de cette enquête.

Nous avons d'abord stratifié la population selon les neuf provinces à l'exception du Québec: dans le cas de l'Ontario, la province a été divisée en trois grandes régions: le Nord-Est, l'Est et le Sud. Ensuite, nous avons choisi des divisions de recensement selon certains critères sociaux et géographiques. A priori, il était important de couvrir l'ensemble du territoire de chaque province, parce que d'une région à l'autre les conditions de vie française peuvent être très différentes. Ensuite, il était nécessaire de répartir les répondants pour représenter des situations où les francophones sont minoritaires, d'autres où ils sont paritaires et d'autres où ils sont majoritaires, le poids démographique étant constamment présenté 
pour expliquer le degré de vitalité ethnolinguistique. Par après, nous avons choisi aléatoirement les institutions scolaires et les répondants. Plus de soixantequinze institutions à travers le Canada ont participé à cette enquête. L'échantillon final retenu regroupait 3801 répondants et 758 de ces répondants vivaient dans des foyers exogames (tableaux 4 et 5 ).

\section{TABLEAU 4}

Répartition des répondants selon la province de résidence échantillon, Canada français (1990).

\begin{tabular}{lrr}
\hline & \multicolumn{2}{c}{$\begin{array}{c}\text { Échantillon } \\
\text { retenu }\end{array}$} \\
\cline { 2 - 3 } Provinces & Nombre & $\%$ \\
Colombie-Britannique & 73 & 1,9 \\
Alberta & 255 & 6,7 \\
Saskatchewan & 153 & 4,0 \\
Manitoba & 330 & 8,7 \\
Ontario & 1446 & 38,0 \\
Nouveau-Brunswick & 1069 & 28,1 \\
Nouvelle-Écosse & 344 & 9,1 \\
Île-du-Prince-Edouard & 94 & 2,5 \\
Terre-Neuve & 37 & 1,0 \\
Total & 3801 & 100,0 \\
& & \\
\hline
\end{tabular}

SOURCES $^{13}$

${ }^{13}$ Roger Bernard, Un avenir incertain, op. cit., p. 246. Comme nous pouvons le constater, près de mille cinq cents répondants demeuraient en Ontario et ces répondants de l'Ontario français représentaient $38 \%$ de l'échantillon global. Le portrait que nous allons tracer des familles exogames francophones de l'ensemble de la francophonie canadienne hors Québec est problablement très près de celui des foyers mixtes de l'Ontario français; en effet, il y a tout lieu de croire que les tendances observées, en regard de la transmission de la langue maternelle et de l'usage du français et de l'anglais à l'intérieur du milieu familial, sont fortement influencées par les jeunes Franco-Ontariens qui forment le noyau principal de l'échantillon. 
3.1.2. L'ECHANTILLON DES FAMILLES EXOGAMES FRANCOPHONES

Comme l'indique le tableau 5 , un jeune francophone sur cinq qui a répondu à notre questionnaire provient d'une famille exogame. Cette proportion globale masque une réalité très différente d'une région à l'autre: en milieu très minoritaire, quatre répondants sur douze sont issus d'une famille exogame, alors que cette proportion est d'un sur douze dans les milieux majoritaires. En reconnaissant le fait que le taux d'exogamie est tributaire du poids démographique de la communauté, il n'est pas surprenant de constater que les taux d'exogamie des jeunes répondants du Québec sont à peu près les mêmes que ceux des jeunes provenant des milieux francophones majoritaires hors Québec. De plus, les données montrent clairement que la proportion de familles exogames dont le père est francophone et la mère anglophone est sensiblement la même que celle dont la mère est francophone et le père anglophone, indépendamment de la province de résidence.

Il est crucial à ce moment de préciser que l'échantillon de l'enquête regroupe seulement des jeunes francophones qui fréquentaient des écoles ou des classes de langue française. Conséquemment, nous pourrions probablement affirmer que les familles exogames que nous avons retenues pour la présente analyse sont des familles exogames à tendance française; elles ont les caractéristiques suivantes:

1) un des parents est de langue maternelle francaise;

2) l'enfant (répondant) a le français ou le français et l'anglais comme langue maternelle; 
3) l'enfant (répondant) fréquente une école française ou une classe de français.

\section{TABLEAU 5}

Taux d'endogamie et d'exogamie des parents des jeunes francophones selon l'indice de contact régional échantillon, Canada français, 1990.

\begin{tabular}{|c|c|c|c|c|c|c|}
\hline \multirow[b]{2}{*}{$\begin{array}{l}\text { Indice de } \\
\text { contact régional }\end{array}$} & \multirow{2}{*}{$\begin{array}{l}\text { Endogamie } \\
\text { Père françals } \\
\text { Mère française }\end{array}$} & \multicolumn{5}{|c|}{ Exogamie } \\
\hline & & Total & $\begin{array}{l}\text { Père anglais } \\
\text { Mère françalse }\end{array}$ & $\begin{array}{l}\text { Mère anglaise } \\
\text { Père français }\end{array}$ & Autres & $\begin{array}{c}\text { Total } \\
(\mathrm{N})\end{array}$ \\
\hline Moins de $5 \%$ & 65,0 & 35,0 & 16,1 & 14,2 & 4,7 & (709) \\
\hline 5 à $19 \%$ & 79,8 & 20,2 & 9,1 & 8,4 & 2,7 & $(882)$ \\
\hline 20 à $39 \%$ & 79,3 & 20,7 & 10,0 & 9,6 & 1,1 & (962) \\
\hline 40 à $59 \%$ & 85,1 & 14,9 & 7,6 & 6,3 & 1,0 & $(576)$ \\
\hline $60 \%$ et plus & 91,2 & 8,7 & 3,9 & 4,1 & 0,7 & (536) \\
\hline Total & 79,3 & 20,7 & 9,7 & 8,9 & $2,1(3$ & $3665)$ \\
\hline Québec & 89,6 & 10,4 & 3,0 & 2,7 & 4,7 & $3212)$ \\
\hline
\end{tabular}

SOURCE ${ }^{14}$

Note: L'indice de contact régional (ICR) représente la proportion de francophones dans la division de recensement du répondant.

Les familles exogames à tendance anglaise ne sont pas représentées dans l'échantillon de l'enquête; à partir de ces données, il ne sera pas possible d'établir le véritable impact de l'exogamie en regard de la continuité linguistique des communautés francophones minoritaires. Si la recherche avait regroupé l'ensemble des enfants francophones et anglophones issus des mariages exogames, il est certain que la prépondérance de l'anglais serait encore beaucoup plus évidente que celle que nous avons observée, et

${ }^{14}$ Québec, échantillon, Conseil de la langue française, 1990; Roger Bernard, ibid., p. 73. 
que la place réservée au français serait encore plus restreinte que celle que nous avons notée.

Le portrait de l'exogamie que nous allons tracer et les enjeux linguistiques et culturels que nous allons dévoiler sont ceux que nous retrouvons à l'intérieur même des communautés francophones et acadiennes. Ce tableau sera tracé à partir d'un sous-échantillon regroupant 758 familles mixtes réparties comme suit: 355 ont un père anglais et une mère française, 325 ont une mère anglaise et un père français et 78 ont un parent francophone et un parent allophone (tableau 6).

\subsubsection{LES TESTS D'HYPOTHESES}

Selon les besoins et la situation, nous avons utilisé deux tests d'hypothèses différents. Dans les cas où nous avions à comparer des pourcentages à l'intérieur d'un même échantillon (est-ce que la proportion qui utilise l'anglais est la même que celle qui utilise le français?), nous avons eu recours au test d'hypothèse sur des pourcentages appliqué à un échantillon. Si nous avions à comparer deux pourcentages tirés de deux échantillons différents (est-ce que la mère francophone réussit mieux que le père francophone à transmettre le français comme langue maternelle unique?), nous avons utilisé le test d'hypothèse sur des pourcentages appliqué à deux échantillons. Les énoncés des différentes hypothèses, les pourcentages comparés, les seuils de signification et les résultats des tests d'hypothèses se trouvent en annexe ${ }^{15}$.

${ }^{15}$ Nous signalons sous forme abrégée ces hypothèses dans le texte; ainsi $(\mathrm{H} 7)$ renvole à l'hypothèse 7 . Pour les détails techniques reliés aux calculs nécessaires pour effectuer un test d'hypothèse sur un pourcentage, il faut consulter un manuel de statistiques. 
3.2. LA FORCE D'ATTRACTION RESPECTIVE DES LANGUES FRANÇAISE ET ANGLAISE

À la question «est-ce que la force d'attraction de l'anglais comme langue maternelle est supérieure à celle du français pour les enfants des mariages mixtes?», force est de répondre, non! À deux exceptions près, les langues anglaise et française se trouvent sur un pied d'égalité en tant que langues maternelles transmises en situation d'exogamie au Canada francophone. La tendance à la bilinguisation de la langue maternelle est nette, et conséquemment nous voyons poindre un nouveau phénomène linguistique et culturel: des jeunes qui ont deux langues maternelles et qui pour la plupart se considèrent comme d'authentiques et véritables bilingues.

En effet, l'analyse des données démontre clairement que la majorité des enfants des familles exogames francophones identifie à la fois l'anglais et le français comme langues maternelles $(52,6 \%)$ et que les autres se répartissent à peu près également entre la langue maternelle française et la langue maternelle anglaise, soit respectivement $22,4 \%$ et $23,9 \%$ (tableau 6). Contrairement aux tendances notées lors de l'étude de presque tous les facteurs linguistiques liés à l'exogamie en situation minoritaire, nous pouvons conclure, lorsque nous considérons l'ensemble des familles mixtes où se trouve un parent francophone, que la force d'attraction de l'anglais comme langue maternelle n'est pas supérieure à celle du français. En considérant toutes les familles exogames francophones qui regroupent un parent francophone et, soit un parent anglophone, soit un parent allophone, la proportion des enfants qui choisissent l'anglais comme langue maternelle unique 
n'est pas plus élevée que la proportion des enfants qui choisissent le français comme langue maternelle unique $(\mathrm{H} 7)$.

TABLEAU 6

Transmission de la langue maternelle chez les jeunes francophones qui vivent différentes situations d'exogamie

Canada français, 1990.

\begin{tabular}{lcccc}
\hline $\begin{array}{l}\text { Langue } \\
\text { maternelle }\end{array}$ & $\begin{array}{l}\text { Père anglais } \\
\text { Mère française }\end{array}$ & $\begin{array}{c}\text { Mère anglaise } \\
\text { Père français }\end{array}$ & $\begin{array}{l}\text { Parent fran. } \\
\text { Parent allo. }\end{array}$ & Total \\
Français & & & & \\
Français/Anglais & 24,8 & 17,2 & 33,3 & 22,4 \\
Anglais & 54,4 & 53,5 & 41,0 & 52,6 \\
Autres & 20,8 & 29,2 & 15,4 & 23,9 \\
& - & - & 10,3 & 1,1 \\
Total \% & $100 \%$ & $100 \%$ & $100 \%$ & $100 \%$ \\
n & 355 & 325 & 78 & 758 \\
\hline
\end{tabular}

SOURCES $^{16}$

Il en va de même dans les familles exogames qui regroupent seulement des parents anglophones et francophones (elles représentent $90 \%$ des cas d'exogamie), la force d'attraction de l'anglais n'est pas plus grande que la force d'attraction du français même s'il semble qu'une proportion plus élevée d'enfants choisissent l'anglais plutôt que le français comme langue maternelle unique, soit $24,8 \%$ en comparaison de $21,2 \%(\mathrm{H} 6)$. C'est le même phénomène, mais à l'inverse, dans les familles exogames qui regroupent une mère francophone et un père anglophone: la force d'attraction du français n'est pas plus grande que la force d'attraction de l'anglais même s'il semble qu'une proportion plus élevée d'enfants choisissent le français plutôt que l'anglais

${ }^{16}$ Roger Bernard, Un avenir incertain, op. cit., p. 74. 
comme langue maternelle unique, soit $24,8 \%$ en comparaison de $20,8 \%$ (H9).

Deux différences sont significatives lorsque nous considérons la force d'attraction de l'anglais en regard du français comme langue maternelle unique. La première est celle des cas d'exogamie entre francophones et allophones (seulement $10 \%$ de l'ensemble des situations d'exogamie) où la force d'attraction du français comme langue maternelle unique dépasse largement la force d'attraction de l'anglais, $33,3 \%$ en comparaison de $15,4 \%$, et la différence est significative (H8). Il est fort probable que le fait que le français soit reconnu comme langue officielle joue un rôle dans l'explication de cette prédominance du français comme langue maternelle unique lorsque l'exogamie réunit un francophone et un allophone. La deuxième différence significative est celle des cas d'exogamie entre un père francophone et une mère anglophone où la force d'attraction de l'anglais comme langue maternelle unique dépasse largement la force d'attraction du français, $29,2 \%$ en comparaison de $17,2 \%$, et la différence est significative (H10). Cependant, lorsque nous considérons l'ensemble des situations d'exogamie, ces différences s'annullent et la force d'attraction de l'anglais comme langue maternelle n'est pas supérieure à la force d'attraction du français.

\subsection{LA FORCE DE RETENTION DES PARENTS RESPECTIFS}

À la question «est-ce que les mères réussissent mieux que les pères à transmettre la langue maternelle aux enfants dans les familles mixtes?», la réponse est oui, lorsqu'il s'agit de la langue maternelle unique. Nous devons cependant rappeler que la 
majorité des enfants identifie à la fois l'anglais et le français comme langues maternelles, et que, dans les familles exogames où la mère est francophone (et le père anglophone), la proportion des enfants qui identifiera à la fois l'anglais et le français comme langues maternelles sera sensiblement la même que celle dans les familles exogames où le père est francophone (et la mère anglophone), soit 54,5\% comparativement à $53,5 \%(\mathrm{H} 2)$.

En revenant au cas de la langue maternelle unique, nous pouvons affirmer que dans les familles exogames où la mère est francophone, la proportion des enfants qui identifiera la français comme langue maternelle unique sera plus élevée que dans les familles exogames où le père est francophone (H1). La même situation existe du côté des anglophones: dans les familles exogames où la mère est anglophone, la proportion des enfants qui identifiera l'anglais comme langue maternelle unique sera plus élevée que dans les familles exogames où le père est anglophone ( $\mathrm{H} 3)$.

Lorsque les enfants francophones des mariages mixtes choisissent une langue maternelle unique, ils sont plus nombreux à choisir la langue de la mère que celle du père, qu'il s'agisse de parents anglophones ou de parents francophones. Donc, dans le cas d'une langue maternelle unique, les mères réussissent mieux que les pères à transmettre leur langue maternelle aux enfants de foyers exogames. Toutefois, il est bon de rappeler que la majorité des enfants ne tranche pas entre les deux langues: ils identifient à la fois l'anglais et le français comme langues maternelles.

Maintenant, passons à des sous-questions. En regard de la transmission de la langue maternelle, estce que les mères anglophones réussissent mieux que 
les mères francophones? En d'autres mots, est-ce que la force de rétention des mères anglophones en ce qui a trait à la transmission de la langue maternelle anglaise est supérieure à la force de rétention des mères francophones par rapport à la transmission de la langue maternelle française? Est-ce que la force de rétention des pères anglophones est supérieure à la force de rétention des pères francophones?

Les résultats des tests d'hypothèses démontrent clairement que la proportion des enfants qui ont l'anglais comme langue maternelle unique dans les familles exogames où la mère est anglophone n'est pas plus élevée que la proportion des enfants qui ont le français comme langue maternelle unique dans les familles exogames où la mère est francophone. Donc, la force de rétention des mères anglophones en regard de la transmission de l'anglais comme langue maternelle n'est pas plus élevée que la force de rétention des mères francophones en regard de la transmission du français comme langue maternelle (H4). Il est essentiel ici de rappeler que l'échantillon regroupe des sujets francophones qui fréquentent des classes ou des écoles de langue française. Les jeunes anglophones et les jeunes francophones issus des mariages exogames mais qui ne fréquentent pas des écoles françaises ne sont pas considérés dans cette étude.

Il en va ainsi des pères: la proportion des enfants qui ont l'anglais comme langue maternelle unique dans les familles exogames où le père est anglophone n'est pas plus élevée que la proportion des enfants qui ont le français comme langue maternelle unique dans les familles exogames où le père est francophone. Par conséquent, la force de rétention des 
pères anglophones en regard de la transmission de l'anglais comme langue maternelle n'est pas supérieure à la force de rétention des pères francophones en regard de la transmission du français comme langue maternelle (H5).

\section{RÉCAPITULATION}

En situation d'exogamie au Canada français (excluant le Québec), les données se résument comme suit en ce qui a trait à la transmission de la langue maternelle:

1. une tendance définitive à la bilinguisation de la langue maternelle: les enfants francophones d'un mariage exogame identifient en majorité à la fois l'anglais et le français comme langues maternelles;

2. lorsque nous considérons la transmission de la langue maternelle unique, nous ne pouvons pas parler de phénomène d'anglicisation: l'anglais et le français se retrouvent sur le même pied à l'intérieur des familles exogames francophones;

3 . les mères réussissent mieux que les pères à transmettre la langue maternelle unique, qu'il s'agisse d'anglophones ou de francophones;

4. les mères anglophones ne réussissent pas mieux que les mères francophones à transmettre leur langue maternelle respective et il en est de même pour les pères anglophones en regard des pères francophones. 
4. Langue d'usage et langue principale dans les FOYERS EXOGAMES FRANCOPHONES

4.1. LA FORCE D'ATTRACTION RESPECTIVE DES LANGUES ANGLAISE ET FRANÇAISE

À la question, «est-ce que la force d'attraction de l'anglais comme langue principale est supérieure à celle du français pour les enfants des mariages mixtes?», force est de répondre, oui! Les données montrent deux grandes tendances, la première étant liée à la bilinguisation et la deuxième, à l'anglicisation. À une exception près, la force d'attraction de l'anglais comme langue principale unique est supérieure à celle du français.

Mais c'est d'abord la bilinguisation qui saute aux yeux: plus de la moitié des jeunes francophones issus de mariages mixtes déclarent à la fois l'anglais et le français comme langues principales (langue dans laquelle les jeunes se sentent le plus à l'aise), et c'est dans les familles mixtes regroupant un parent francophone et un parent allophone que cette proportion est la plus élevée (57,7\%) (tableau 7). Lorsque nous considérons les choix uniques liés à l'anglais ou au français, c'est la tendance à l'anglicisation qui apparaît clairement; en effet, si nous retenons l'ensemble des familles exogames, un tiers des enfants qui en sont issus identifient l'anglais comme langue principale unique, contre une faible minorité $(12,9 \%)$ qui se dit plus à l'aise en français et la différence est significative: la force d'attraction de l'anglais comme langue principale unique est supérieure à la force d'attraction du français (H15). Il en est ainsi dans les familles exogames qui regroupent seulement des parents anglophones et francophones: la force d'attraction de l'anglais en regard du 
français ne dément pas, qu'il s'agisse de la mère qui soit francophone $(\mathrm{H} 16)$ ou du père $(\mathrm{H} 17)$. La seule différence qui n'est pas statistiquement significative est celle des cas d'exogamie entre francophones et allophones où la force d'attraction de l'anglais et du français en tant que langue principale unique se retrouve sur un pied d'égalité, même si la proportion de jeunes francophones qui identifie l'anglais semble plus élevée que celle qui identifie le français, 19,2\% contre $14,1 \%(\mathrm{H} 19)$. Nous pouvons donc affirmer que, d'un côté, la force d'attraction de l'anglais est supérieure à celle du français (il y a là une forme d'anglicisation) et, d'un autre côté, les parents francophones ne réussissent pas très bien à imposer le français comme langue principale unique, c'est-àdire que leur force de rétention du français est inférieure à celle des parents anglophones en regard de la rétention de l'anglais. Lorsque les parents francophones réussissent à maintenir le français, c'est habituellement par le biais du bilinguisme: la majorité des enfants francophones de foyers exogames identifient à la fois l'anglais et le français comme langues principales et cette tendance à la bilinguisation se retrouve immanquablement dans toutes les différentes situations d'exogamie. Toutefois, nous devons noter que la proportion d'enfants qui identifient à la fois l'anglais et le français comme langues principales est significativement plus élevée dans les mariages exogames où la mère est francophone que dans les mariages exogames où le père est francophone $(\mathrm{H} 13)$; dans les autres situations d'exogamie, eu égard au comportement bilingue, les différences ne sont pas significatives ( $\mathrm{H} 20, \mathrm{H} 21)$. 
TABLEAU 7

Langue principale des jeunes francophones qui vivent différentes situations d'exogamie, Canada français, 1990.

\begin{tabular}{|c|c|c|c|c|}
\hline $\begin{array}{l}\text { Langue } \\
\text { principale }\end{array}$ & $\begin{array}{l}\text { Père anglais } \\
\text { Mère française }\end{array}$ & $\begin{array}{l}\text { Mère anglaise } \\
\text { Père français }\end{array}$ & $\begin{array}{l}\text { Parent francophone } \\
\text { Parent allophone }\end{array}$ & Total \\
\hline Français & 10,4 & 14,2 & 14,1 & 12,4 \\
\hline Français/Anglais & 58,3 & 49,8 & 57,7 & 54,6 \\
\hline Anglais & 30,4 & 35,4 & 19,2 & 31,4 \\
\hline Autres & 0,8 & 0,6 & 9,0 & 1,4 \\
\hline Total \% & $100 \%$ & $100 \%$ & $100 \%$ & $100 \%$ \\
\hline $\mathrm{n}$ & 355 & 325 & 78 & 758 \\
\hline
\end{tabular}

SOURCE $^{17}$

\subsection{LA FORCE DE RETENTION DES PARENTS RESPECTIFS}

À la question «est-ce que les mères réussissent mieux que les pères à transmettre aux enfants dans les familles mixtes leur langue maternelle respective comme langue principale unique?», la réponse est non.

Les mères francophones ne réussissent pas mieux que les pères francophones à retenir le français comme langue principale unique: la proportion des enfants francophones de foyers exogames qui identifient le français comme langue principale unique n'est pas plus élevée dans les familles où la mère est francophone que dans celles où le père est francophone (H11). Il en va de même du côté anglophone. Les mères anglophones ne réussissent pas mieux que les pères anglophones à imposer l'anglais comme langue principale unique: la proportion des enfants qui identifient l'anglais comme langue principale unique n'est pas plus élevée dans les familles où la

${ }^{17}$ Ibid., p. 75. 
mère est anglophone que dans celle où le père est anglophone (H12).

Nous passons maintenant aux sous-questions. Estce que les mères anglophones réussissent mieux que les mères francophones à établir leur langue maternelle respective en tant que langue principale unique des enfants? Est-ce que la force de rétention des pères anglophones est supérieure à la force de rétention des pères francophones en ce qui a trait à la langue principale unique des enfants? Les résultats des tests d'hypothèse démontrent clairement la supériorité de la force de rétention de la mère anglophone pour le maintien de l'anglais comme langue principale unique des enfants francophones en regard de la force de rétention de la mère francophone pour le maintien du français (H18). Il en est ainsi du côté des pères: nette supériorité de la force de rétention du père anglophone en regard du père francophone pour le maintien de leur langue respective (H19). La supériorité de la force de rétention des parents anglophones ne fait aucun doute en regard de la force de rétention des parents francophones $(\mathrm{H} 16, \mathrm{H} 17, \mathrm{H} 18, \mathrm{H} 19)$ lorsque nous analysons les données relatives à la langue principale.

\section{RÉCAPITULATION}

En situation d'exogamie au Canada français (excluant le Québec), les données se résument comme suit en ce qui a trait à la langue principale des jeunes francophones issus des familles exogames francophones:

1. une nette tendance à la bilinguisation de la langue principale: les enfants francophones issus 
d'un mariage exogame identifient en majorité à la fois l'anglais et le français comme langues principales (langue dans laquelle l'enfant se sent le plus à l'aise);

2. lorsque nous considérons la langue principale unique, nous pouvons parler d'un phénomène d'anglicisation: la force d'attraction de l'anglais dépasse nettement la force d'attraction du français;

3. les mères francophones ne réussissent pas mieux que les pères francophones à retenir le français comme langue principale unique; il en est de même pour les mères et les pères anglophones en regard de l'anglais;

4. les mères anglophones réussissent mieux que les mères francophones à retenir leur langue respective en tant que langue principale unique des jeunes francophones; il en est ainsi pour les pères anglophones en regard des pères francophones.

\subsection{LANGUE D'USAGE À LA MAISON DANS LES FOYERS EXOGAMES} FRANCOPHONES

Il ressort clairement d'après les données du tableau 8 , que la force d'attraction de l'anglais est très nette et supérieure au français en regard de la langue le plus souvent parlée à la maison dans les familles exogames. En effet, près de trois jeunes francophones sur quatre identifient l'anglais comme étant la langue officielle le plus souvent parlée à la maison, comparativement à une minorité $(29,7 \%)$ qui déclare le français comme langue d'usage ( $\mathrm{H} 22)$. Dans toutes les situations d'exogamie, l'anglais s'impose immanquablement comme langue du foyer $(\mathrm{H} 23$, $\mathrm{H} 28, \mathrm{H} 29$ ). 
TABLEAU 8

Langue officielle parlée le plus souvent à la maison par des jeunes francophones qui vivent différentes situations d'exogamie

Canada français, 1990.

\begin{tabular}{lcccc}
\hline $\begin{array}{l}\text { Langue } \\
\text { d'usage à la } \\
\text { maison }\end{array}$ & $\begin{array}{l}\text { Père anglais } \\
\text { Mère française }\end{array}$ & $\begin{array}{l}\text { Mère anglaise } \\
\text { Père français }\end{array}$ & $\begin{array}{l}\text { Parent francophone } \\
\text { Parent allophone }\end{array}$ & Total \\
Français & 30,3 & 26,4 & 40,5 & \\
Anglais & 69,7 & 73,6 & 59,5 & 70,3 \\
& & & & \\
Total \% & $100 \%$ & $100 \%$ & $100 \%$ & $100 \%$ \\
n & 346 & 314 & 74 & 734 \\
\hline
\end{tabular}

SOURCE $^{18}$

La force de rétention des parents anglophones est réellement supérieure à celle des parents francophones $(\mathrm{H} 24, \mathrm{H} 25)$; même dans les foyers exogames réunissant un parent francophone et un parent allophone, l'anglais s'impose $(59,3 \%)$, mais le français occupe une place plus importante $(40,5 \%)$ que dans les autres situations d'exogamie ( $\mathrm{H} 23)$. L'anglicisation ne fait aucun doute lorsque nous considérons la langue d'usage à la maison dans les foyers exogames, mais le français est retenu comme langue parlée le plus souvent à la maison dans un tiers des familles. Il faut à ce moment rappeler que l'échantillon regroupait seulement des jeunes francophones qui fréquentaient des écoles ou des classes de langue française. Si la recherche avait regroupé l'ensemble des enfants francophones et anglophones issus des mariages exogames, il est certain que la prépondérance de l'anglais serait encore plus évidente et que la place dévolue au français, plus restreinte. Nous pouvons done voir à l'œuvre des processus d'anglicisation dans le nouvel espace familial exogame. Toutefois, il faut aussi rap-

${ }^{18}$ Ibid., p. 76. 
peler que l'exogamie, la minorisation et la dispersion sont des phénomènes concomitants et que nous ne pouvons pas établir clairement le poids respectif de ces différents phénomènes démographiques pour expliquer les taux d'anglicisation et d'assimilation culturelle. Nous pouvons cependant soutenir que l'exogamie, dans le contexte actuel de minorisation et de dispersion des francophones au Canada français, provoque l'anglicisation du milieu familial d'une proportion de plus en plus importante de jeunes francophones qui fréquentent les écoles de langue française.

À la question, «est-ce que les mères réussissent mieux que les pères à imposer dans le milieu familial leur langue maternelle respective comme langue d'usage?» la réponse est non! Les mères francophones ne réussissent pas mieux que les pères francophones à imposer le français comme langue d'usage à la maison: la proportion de jeunes francophones qui ont identifié le français comme langue d'usage à l'intérieur de la maison n'est pas plus élevée dans les familles mixtes où la mère est francophone $(30,3 \%)$ que dans celles où le père est francophone $(26,4 \%)$ (H26). Il en est ainsi pour les mères anglophones en regard des pères anglophones: la proportion est sensiblement la même dans les deux cas ( $\mathrm{H} 27)$.

\section{RÉCAPITULATION}

En ce qui a trait à la langue d'usage dans les familles exogames francophones, les données se résument comme suit:

1. une nette tendance à l'anglicisation en ce qui a trait à la langue d'usage dans les foyers exogames; 
2. les mères francophones ne réussissent pas mieux que les pères francophones à retenir le français comme langue d'usage dans le milieu familial exogame.

\section{Conclusion}

Dans le contexte culturel et religieux de la société canadienne-française, l'exogamie est à la fois impensable et impraticable; elle s'apparente à la transgression d'un tabou. Le passage d'un monde rural et agricole à un monde urbain et industriel provoque la minorisation et l'hétérogénéité, conditions qui encouragent l'expansion de l'exogamie comme forme d'organisation familiale. Aujourd'hui, l'exogamie est une réalité avec laquelle les communautés francophones et acadiennes doivent composer; de fait, nous assistons à l'institutionnalisation de l'exogamie. Depuis 1971, les tendances démographiques ne démordent pas: en situation très minoritaire, l'exogamie est omniprésente, alors qu'en situation majoritaire, elle est quasi absente.

Les recherches indiquent clairement que les taux d'exogamie et de transferts linguistiques vers l'anglais augmentent à travers le temps et les générations et qu'ils sont fortement corrélés. L'exogamie est certes une cause importante de l'assimilation, mais il ne s'agit pas de l'unique cause.

Les données de l'enquête auprès des jeunes francophones canadiens (excluant les jeunes Québécois) montrent une nette tendance à la bilinguisation lorsque nous examinons les processus de transmission de la langue maternelle à l'intérieur des foyers exogames francophones: une majorité d'enfants ont 
à la fois l'anglais et le français comme langue maternelle et les proportions ne sont pas différentes selon que la mère soit francophone plutôt que le père. Lorsque nous considérons la transmission d'une langue maternelle unique, l'anglais et le français sont sur un pied d'égalité, et les mères réussissent mieux que les pères à la transmettre, indépendamment qu'il s'agisse d'anglophones ou de francophones.

La force d'attraction de l'anglais comme langue principale (langue dans laquelle les jeunes se sentent plus à l'aise) est supérieure à celle du français pour les enfants des mariages mixtes francophones. Les données dévoilent deux tendances: l'une est liée à la bilinguisation des comportements linguistiques et l'autre à l'anglicisation, et les mères francophones ne réussissent pas mieux que les pères francophones à retenir pour les enfants le français comme langue principale unique.

En ce qui a trait à la langue d'usage à la maison, la force d'attraction de l'anglais est très nette en regard du français: en effet, dans toutes les situations de familles exogames francophones, l'anglais s'impose immanquablement comme langue du foyer, et les mères francophones ne réussissent pas mieux que les pères francophones à retenir l'usage du français. 


\section{ANNEXX 1}

HYPOTHĖSES EN REGARD DE LA TRANSMISSION DE LA LANGUE MATERNELLE (tableau 6)

\section{HYPOTHËSE 1}

Dans les familles exogames où la mère est francophone, la proportion des enfants qui identifieront le français comme langue maternelle sera plus élevée que dans les familles exogames où le père est francophone.

H1: $17,2 \%<24,8 \% ; \alpha=0,05$

Acceptée

\section{HYPOTHËSE 2}

Dans les familles exogames où la mère est francophone, la proportion des enfants qui identifieront à la fois l'anglais et le français comme langues maternelles ne sera pas la même que dans les familles exogames où le père est francophone.

H1: $53,5 \% \neq 54,5 \% ; \alpha=0,05$

Rejetée

\section{HYPOTHĖSE 3}

Dans les familles exogames où la mère est anglophone, la proportion des enfants qui identifieront l'anglais comme langue maternelle sera plus élevée que dans les familles exogames où le père est anglophone.

H 1: $29,2 \%>20,8 \% ; \alpha=0,05$

\section{Acceptée}

\section{HYPOTHĖSE 4}

La proportion des enfants qui ont l'anglais comme langue maternelle dans les familles exogames où la mère est anglophone est plus élevée que la proportion des enfants qui ont le français comme langue maternelle dans les familles exogames où la mère est francophone.

H1: $24,8 \%<29,2 \% ; \alpha=0,05$

Rejetée 


\section{HYPOTHÈSE 5}

La proportion des enfants qui ont l'anglais comme langue maternelle dans les familles exogames où le père est anglophone est plus élevée que la proportion des enfants qui ont le français comme langue maternelle dans les familles exogames où le père est francophone.

H1: $20,8 \%>17,2 \% ; \alpha=0,05$

Rejetée

HYPOTHESE 6

Dans les familles exogames qui regroupent des parents anglophones et francophones seulement, la proportion des enfants qui choisissent l'anglais comme langue maternelle sera plus élevée que la proportion des enfants qui choisissent le français.

$H 1: 21,2 \%<24,8 \% ; \alpha=0,05$

Rejetée

HYPOTHĖSE 7

Dans toutes les familles exogames qui regroupent un parent francophone, la proportion des enfants qui choisissent l'anglais comme langue maternelle sera plus élevée que la proportion des enfants qui choisissent le français.

H1: $22,4 \%<23,9 \% ; \alpha=0,05$

Rejetée

HYPOTHËSE 8

Dans les familles exogames qui regroupent un parent francophone et un parent allophone, la proportion des enfants qui choisissent le français comme langue matemelle unique sera supérieure à la proportion des enfants qui choisissent l'anglais.

H1: $33,3 \%>15,4 \% ; \alpha=0,05$

Acceptée 
HYPOTHESE 9

Dans les familles exogames regroupant un père anglophone et une mère francophone, la proportion des enfants qui identifieront le français comme langue maternelle sera supérieure à la proportion qui identifiera l'anglais comme langue maternelle.

H1: $24,8 \%>20,8 \% ; \alpha=0,05$

Rejetée

HYPOTHĖSE 10

Dans les familles exogames regroupant un père francophone et une mère anglophone, la proportion des enfants qui identifieront l'anglais comme langue maternelle sera supérieure à la proportion qui identifiera le français comme langue maternelle.

H1: $17,2 \%<29,2 \% ; \alpha=0,05$

Acceptée 


\section{ANNEXE 2}

HYPOTHESES EN REGARD DE LA LANGUE PRINCIPALE (tableau 7)

\section{HYPOTHESE 11}

La proportion des enfants qui identifieront le français comme langue principale est plus élevée dans les familles mixtes où la mère est francophone que dans les familles mixtes où le père est francophone.

HI: $10,4 \%<14,2 \% ; \alpha=0,05$

Rejetée

\section{HYPOTHËSE 12}

La proportion des enfants qui identifieront l'anglais comme langue principale est plus élevée dans les familles mixtes où la mère est anglophone que dans les familles mixtes où le père est anglophone.

H1: $30,4 \%<35,4 \% ; \alpha=0,05$

Rejetée

\section{HYPOTHËSE 13}

La proportion des enfants qui identifient à la fois l'anglais et le français comme langues principales est plus élevée dans les mariages mixtes où la mère est francophone que dans les mariages mixtes où le père est francophone.

Hl: $58,3 \%>49,8 \% ; \alpha=0,05$

Acceptée

\section{HYPOTHËSE 14}

Dans les mariages exogames qui réunissent un parent francophone et un parent allophone, la proportion des enfants qui identifieront l'anglais comme langue principale sera supérieure à celle qui identifiera le français comme langue principale.

H1: $14,1 \%<19,2 \% ; \alpha=0,05$

Rejetée 


\section{HYPOTHĖSE 15}

Dans l'ensemble des familles exogames qui réunissent un parent francophone et un parent allophone ou anglophone, la proportion des enfants qui identifieront l'anglais comme langue principale sera supérieure à celle qui identifiera le français comme langue principale.

$\mathrm{H} 1: 12,4 \%<31,4 \% ; \alpha=0,05$

Acceptée

HYPOTHESE 16

Dans les familles exogames regroupant un père anglophone et une mère francophone, la proportion des enfants qui identifieront l'anglais comme langue principale sera supérieure à celle qui identifiera le français comme langue principale.

H1: $10,4 \%<30,4 \% ; \alpha=0,05$

Acceptée

HYPOTHĖSE 17

Dans les familles exogames regroupant un père francophone et une mère anglophone, la proportion des enfants qui identifieront l'anglais comme langue principale sera supérieure à celle qui identifiera le français comme langue principale.

H1: $14,2 \%<35,4 \% ; \alpha=0,05$

Acceptée

HYPOTHĖSE 18

La proportion des enfants qui ont l'anglais comme langue principale dans les familles exogames où la mère est anglophone est plus élevée que la proportion des enfants qui ont le français comme langue maternelle dans les familles exogames où la mère est francophone.

H1: $35,4 \%>10,4 \% ; \alpha=0,05$

Acceptée 
HYPOTHESE 19

La proportion des enfants qui ont l'anglais comme langue principale dans les familles exogames où le père est anglophone est plus élevée que la proportion des enfants qui ont le français comme langue principale dans les familles exogames où le père est francophone.

H1: $30,4 \%>14,2 \% ; \alpha=0,05$

Acceptée

HYPOTHËSE 20

La proportion des enfants qui identifient à la fois l'anglais et le français comme langues principales est plus faible dans les familles exogames où la mère est anglophone et le père francophone que dans les familles exogames qui regroupent un parent francophone et un parent allophone.

H1: $57,7 \%>49,8 \% ; \alpha=0,05$

Rejetée

HYPOTHESE 21

La proportion des enfants qui identifient à la fois l'anglais et le français comme langues principales est plus élevée dans les familles exogames où la mère est francophone et le père anglophone que dans les familles exogames qui regroupent un parent francophone et un parent allophone.

$\mathrm{H} 1: 57,7 \%<58,3 \% ; \alpha=0,05$

Rejetée 


\section{ANNEXE 3}

HYPOTHĖSES EN REGARD DE LA LANGUE D'USAGE AU FOYER (tableau 8)

HYPOTHESES 22, 23, 28, 29

Lorsque nous considérons l'ensemble des situations exogames $(\mathrm{H} 22)$ ou celles qui regroupent un père anglophone et une mère francophone $(\mathrm{H} 28)$, un père francophone et une mère anglophone $(\mathrm{H} 29)$ ou un parent francophone et un parent allophone $(\mathrm{H} 23)$, la proportion des enfants qui identifient l'anglais comme langue d'usage à la maison est toujours plus élevée que celle qui identifie le français comme langue d'usage au foyer.

H22: $\mathrm{H} 1: 29,7 \%<70,3 \% ; \alpha=0,05$

H23: H1: $59,5 \%>40,5 \% ; \alpha=0,05$

H28: H1: $30,3 \%<69,7 \% ; \alpha=0,05$

H29: H1: $26,4 \%<73,6 \% ; \alpha=0,05$

Acceptées

HYPOTHĖSE 24

La proportion des enfants qui identifient l'anglais comme langue d'usage à la maison est plus élevée dans les familles exogames où la mère est anglophone que la proportion des enfants qui identifient le français comme langue d'usage dans les familles exogames où la mère est francophone.

H1: $30,3 \%<73,6 \% ; \alpha=0,05$

Acceptée

HYPOTHĖSE 25

La proportion des enfants qui identifient l'anglais comme langue d'usage à la maison est plus élevée dans les familles exogames où le père est anglophone que la proportion des enfants qui identifient le français comme langue 
d'usage dans les familles exogames où le père est francophone.

H1: $69,7 \%>26,4 \% ; \alpha=0,05$

Acceptée

HYPOTHESE 26

Dans les familles exogames où la mère est francophone, la proportion des enfants qui identifieront le français comme langue d'usage à la maison sera plus élevée que dans les familles exogames où le père est francophone.

H1: $30,3 \%>26,4 \% ; \alpha=0,05$

Rejetée

HYPOTHESE 27

Dans les familles exogames où la mère est anglophone, la proportion des enfants qui identifieront l'anglais comme langue d'usage à la maison sera plus élevée que dans les familles exogames où le père est anglophone.

H1: $69,7 \%<73,6 \% ; \alpha=0,05$

Rejetée 